\title{
Relationships between climate and sperm quality in dairy bull semen: A retrospective analysis
}

\author{
M. Sabés-Alsina, ${ }^{1}$ N. Lundeheim, ${ }^{2}$ A. Johannisson, ${ }^{1}$ M. López-Béjar, ${ }^{3}$ and J. M. Morrell ${ }^{1 *}$ \\ ${ }^{1}$ Division of Reproduction, Department of Clinical Sciences, Swedish University of Agricultural Sciences, SE-75007 Uppsala, Sweden \\ ${ }^{2}$ Department of Animal Breeding and Genetics, Swedish University of Agricultural Sciences, SE-75007 Uppsala, Sweden \\ ${ }^{3}$ Department of Animal Health and Anatomy, Universitat Autònoma de Barcelona, 08193 Bellaterra, Spain
}

\section{ABSTRACT}

The aim of the present study was to make a retrospective analysis of the relationship between climatic factors and sperm quality of frozen-thawed semen from bulls kept in temperate climates. Semen samples from 21 European dairy bulls from 2 countries were collected and cryopreserved in winter, spring, and summer. Sperm quality parameters such as kinematics, morphology, plasma membrane integrity, mitochondrial membrane potential, sperm chromatin structure assay, and reactive oxygen species were analyzed and correlated retrospectively with climate factors recorded by the local meteorological office. This study demonstrated that sperm quality parameters are more likely to be correlated with climate factors 1 or 2 mo before semen collection than in the month of semen collection. During the month of sperm collection, sperm kinematics, DNA fragmentation, and hydrogen peroxide production were the only sperm quality parameters related to climate factors, whereas 1 and 2 mo before sperm collection, normal morphology and additional sperm kinematics, in addition to DNA fragmentation and hydrogen peroxide production, were correlated with climate factors. In conclusion, dairy bull sperm quality is affected by climatic conditions, even in so-called temperate zones. The timing of heat stress during spermatogenesis determines which aspects of sperm quality are likely to be affected. Husbandry conditions for bulls used for semen collection should be adapted to allow the animals' physiological responses for temperature regulation within the scrotum to operate fully, to mitigate the effects of increased temperature and humidity. Extremes of temperature should be avoided.

Received October 12, 2018.

Accepted January 28, 2019.

*Corresponding author: jane.morrell@slu.se
Key words: seasonal effects, kinematics, DNA fragmentation, cattle

\section{INTRODUCTION}

Sperm quality can be described as the ability of the spermatozoa to move from the site of semen deposition to the site of fertilization, to penetrate an oocyte and activate it to develop an embryo. It can be defined in terms of motility, morphology, plasma membrane integrity, metabolic activity, and ability to acrosome react, among others (Mocé and Graham, 2008; Morrell and Rodríguez-Martínez, 2009). All of these sperm characteristics are susceptible to extraneous factors such as climate, level of nutrition, and management practice (Brown, 1994; Snoj et al., 2013; Bhakat et al., 2014), as well as intrinsic factors such as age (Snoj et al., 2013). The assessment of sperm quality parameters in the laboratory is a convenient indirect way of detecting alterations in a male's ability to reproduce, without conducting an AI trial on each ejaculate.

Sperm quality is highly sensitive to even a mild and brief increase in scrotal temperature, which may reflect environmental influences on the delicate stages of spermatogenesis at any time (Januskauskas et al., 1995). Spermatogenesis in bulls takes around $60 \mathrm{~d}$; therefore, alterations in the ejaculate characteristics produced by deleterious events, such as changes in testicular temperature, may not be observed immediately. However, possible spermatogenic cell damage may be seen in the ejaculate 2 to 4 wk after heat stress (Vogler et al., 1993) and sperm quality may not return to normality until 6 to 12 wk later (Amann and Almquist, 1962; Kastelic et al., 1997a; Hansen, 2009). Therefore, the effect of climate on sperm quality may be missed if the sperm quality evaluation is made at an inappropriate time.

The factor of "climate" itself can vary depending on which factor, or combination of factors, is being measured. Thus, both temperature and humidity contribute to the physiological responses triggered to regulate body temperature and these 2 factors can be combined 
as a "temperature-humidity index" (THI) (Bohmanova et al., 2007). Some authors report a negative effect on cow fertility due to heat stress at THI $>72$ and on milk yield if the THI is $\geq 78$ (Gantner et al., 2011). However, other factors may also be involved in the effects of "climate" on sperm quality, such as atmospheric pressure (Verratti et al., 2016) or day length. The latter has a direct effect on reproduction via endocrine regulation, but also has an indirect effect on heat stress by affecting solar radiation (Blackshaw and Blackshaw, 1994). All of these factors should be taken into consideration when discussing the overall effects of climate on sperm quality.

The aim of the present study was to make a retrospective analysis of the relationship between climatic factors and sperm quality of frozen-thawed bovine semen. Correlations were made between frozen-thawed sperm quality and climate factors measured at several time points; namely, during the month of semen collection, 1 mo before semen collection, and 2 mo before semen collection. The study population consisted of bulls from a semen collection center in western Sweden (Valeanu et al., 2015) and another in northern Spain (Sabés-Alsina et al., 2017). These areas are classified as having a temperate climate (Belda et al., 2014); that is, not being excessively hot or cold. The climate of Sweden is milder and drier than other regions at comparable latitudes due to the warming effect of the Gulf Stream (https://visitsweden.com/weather-and -climate/; accessed September 2018). The climate of northern Spain, where the bull station is located, is characterized by having cool summers and mild winters with few extreme temperatures. In addition, there is year-round precipitation (AEMET, www.aemet.es; Weatherbase, www.weatherbase.com; accessed September 2018). Meteorological data were available for the respective regions from recording stations located near the bull stations.

\section{MATERIALS AND METHODS}

\section{Experimental Design}

For the purpose of our research, different sperm quality parameters were analyzed and correlated retrospectively with climate factors recorded by the local meteorological office. Sperm concentration, kinematics, and morphology were determined using the NucleoCounter SP-100 (ChemoMetec A/S, Allerød, Denmark) computer-assisted sperm analysis and microscopy, respectively. Sperm functional parameters such as plasma membrane integrity, mitochondrial membrane potential, sperm chromatin structure assay (SCSA), and reactive oxygen species (ROS) were analyzed using flow cytometry.

\section{Animals and Semen Collection}

Semen samples from 21 dairy bulls from 2 European countries (11 Holstein in Spain; 3 Holstein and 7 Swedish Red in Sweden) were collected during winter (December-January; $\mathrm{n}=21$ ), spring (March-April; $\mathrm{n}=$ 18), and summer (July-August; $n=21$ ). The animals were in the regular collection schedule of the station during these times; that is, an ejaculate was collected at least once per week. One ejaculate was collected per male and season. Unfortunately, no straws were available for the spring time point from 3 of the Spanish bulls. The Spanish bulls were housed in outdoor facilities and thus were exposed to natural climate conditions and photoperiod, whereas the Swedish bulls were housed indoors, with lighting during the working day $(7 \mathrm{~h})$ in winter.

Sperm samples were collected using an artificial vagina. Only fresh semen samples with good mass motility $(>70 \%)$ on visual assessment were extended in a non-egg-yolk extender [Andromed (Minitube, Tiefenbach, Germany) for the Swedish samples; BioXcell (IMV Technologies, l'Aigle, France) for the Spanish samples] and cryopreserved. After thawing, only straws with post-thaw motility $\geq 50 \%$ were retained by the bull center and thus were included in the study.

\section{Climate Factors}

Temperature $\left({ }^{\circ} \mathrm{C}\right)$, humidity $(\%)$, atmospheric pressure $(\mathrm{hPa})$, and visible daylight length $(\mathrm{min} / \mathrm{d})$ from both countries were obtained from Sweden's Meteorological and Hydrological Institute (SMHI, Linköping, Sweden; www.smhi.se; accessed September 2018), and the Agencia Estatal de Meteorología (AEMET, Gijón, Spain; www.aemet.es; accessed September 2018) and the Instituto Geográfico Nacional (Madrid, Spain; www .ign.es, accessed September 2018).

The meteorological station in Sweden was located approximately $23 \mathrm{~km}$ from the bull station, which was located at $58.39^{\circ} \mathrm{N} 13.49^{\circ} \mathrm{E}$. In Spain, the meteorological station was approximately $11 \mathrm{~km}$ from the bull station $\left(43.56^{\circ} \mathrm{N}\right.$ and $\left.-5.70^{\circ} \mathrm{E}\right)$. The mean and maximum $(\max )$ THI (García-Ispierto et al., 2006) were calculated using the following formulas:

$$
\begin{aligned}
& \text { Mean THI }= \\
& 0.8 \times \text { mean } \mathrm{T}+\frac{\text { mean } \mathrm{RH}(\%)}{100} \times(\text { mean } \mathrm{T}-14.4)+46.4 ;
\end{aligned}
$$


$\operatorname{Max} \mathrm{THI}=$

$0.8 \times \max \mathrm{T}+\frac{\text { minimum } \mathrm{RH}(\%)}{100} \times(\max \mathrm{T}-14.4)+46.4 ;$

where $\mathrm{T}=$ temperature $\left({ }^{\circ} \mathrm{C}\right)$ and $\mathrm{RH}=$ relative humidity.

Mean climate factors from the month of sperm collection and 1 mo and 2 mo before collection were correlated with the sperm quality parameters.

\section{Sperm Quality Analysis}

Sperm Thawing and Concentration Determination. One straw $(0.25 \mathrm{~mL})$ was thawed for each bull and season. Straws were thawed in a water bath at $37^{\circ} \mathrm{C}$ for $12 \mathrm{~s}$, according to the laboratory routine. Sperm concentration was determined using the NucleoCounter SP-100 (ChemoMetec A/S). The protocol was modified slightly from the manufacturer's recommendation, using an aliquot $(5 \mu \mathrm{L})$ of sample mixed thoroughly with $500 \mu \mathrm{L}$ of reagent $\mathrm{S} 100$ instead of $50 \mu \mathrm{L}$ and $5 \mathrm{~mL}$, respectively.

Sperm Motility Assessment with ComputerAssisted Sperm Analysis. Sperm kinematics were determined using the SpermVision system (Minitüb, Tiefenbach, Germany) connected to an Olympus BX 51 microscope (Olympus, Tokyo, Japan). A $5-\mu \mathrm{L}$ aliquot of semen was pipetted on to a warmed microscope slide $\left(38^{\circ} \mathrm{C}\right)$, covered with an $18-\times 18-\mathrm{mm}$ coverslip, and analyzed. A minimum of 200 cells per field was analyzed in 8 fields. Total motility (MT, \%), progressive motility (MP , \%), distance average path (DAP, $\mu \mathrm{m})$, distance curved line (DCL, $\mu \mathrm{m})$, distance straight line (DSL, $\mu \mathrm{m})$, average path velocity (VAP, $\mu \mathrm{m} / \mathrm{s})$, curvilinear velocity (VCL, $\mu \mathrm{m} / \mathrm{s})$, straight-line velocity $($ VSL,$\mu \mathrm{m} / \mathrm{s})$, straightness $(\mathbf{S T R}=\mathrm{VSL} / \mathrm{VAP}, \%)$, linearity $(\mathbf{L I N}=\mathrm{VSL} / \mathrm{VCL}, \%)$, wobble $(\mathbf{W O B}=$ VAP/VCL, \%), amplitude of lateral head displacement $(\mathbf{A L H}, \mu \mathrm{m})$, and beat cross frequency $(\mathbf{B C F}, \mathrm{Hz})$ data were collected.

Sperm Morphology Evaluation. Sperm morphology was evaluated as described previously (Morrell et al., 2008). Briefly, air-dried smears were stained using William's staining to assess sperm head shape; 500 sperm were evaluated at $1,000 \times$ magnification by light microscopy (Leitz Microsystems, Wetzlar, Germany). In addition, aliquots $(5 \mu \mathrm{L})$ were fixed with $45 \mu \mathrm{L}$ of formol saline solution for preparation of wet smears. Slides were analyzed using a phase-contrast microscope (Leica Microsystems) at 1,000× magnification, evaluating at least 200 sperm per sample. The percentage of spermatozoa with normal morphology was estimated by deducting the spermatozoa with morphological ab- normalities from the total. Morphology evaluation was carried out by skilled personnel in the Swedish Sperm Reference Laboratory at the Swedish University of Agricultural Sciences.

\section{Flow Cytometry Analysis}

Instruments and Settings. The sperm samples from the Spanish bulls were analyzed using a BD LSR flow cytometer (Becton Dickinson, San Jose, CA) equipped with an argon-ion laser emitting at $488 \mathrm{~nm}$ and a helium-cadmium laser emitting at 325 $\mathrm{nm}$. The flow rate was $35 \mu \mathrm{L} / \mathrm{min}$. For the Swedish bulls, the SCSA samples were run on a FACStarPLUS flow cytometer (Becton Dickinson) equipped with an argon-ion laser emitting at $488 \mathrm{~nm}$. Acquisition was performed using Cellquest (version 3.3; Becton Dickinson) for both cytometers. This software was also used for data analysis, except for analysis of SCSA data, which was performed using FCS Express version 2 (De Novo Software, Thornhill, ON, Canada). In all cases, analysis was restricted to spermatozoa by using a gate in forward scatter (FSC) and side scatter (SSC). For all SCSA samples, the flow rate was adjusted to analyze 200 events/s for all samples.

Plasma Membrane Integrity Assessment. Plasma membrane integrity was assessed using SYBR-14 and propidium iodide (PI) staining (Live-Dead Sperm Viability KIT LIT L-7011; Invitrogen, Eugene, OR). Briefly, aliquots from thawed semen samples were diluted to approximately $2 \times 10^{6}$ spermatozoa $/ \mathrm{mL}$ with buffer B (patent applied for; J. M. Morrell and H. Rodriguez-Martinez). An aliquot of $300 \mu \mathrm{L}$ of the diluted sample was stained with $1.2 \mu \mathrm{L}$ of SYBR-14 (final stain concentration $0.04 \mu \mathrm{M}$ ) and $3 \mu \mathrm{L}$ of PI (final concentration $12 \mu M$ ) and incubated at $37^{\circ} \mathrm{C}$ in the dark for 10 min before cytometric analysis. Green fluorescence of the plasma membrane integrity assay was detected with an FL1 band-pass filter $(530 / 28 \mathrm{~nm})$, and red fluorescence was measured using an FL3 long-pass filter (>670 nm). Compensation was not used.

After cytometric analysis, sperm cells were quantified according to the integrity of the plasma membrane as follows: live spermatozoa with intact plasma membrane (SYBR-14 positive/PI negative), moribund spermatozoa (SYBR-14 intermediate/PI intermediate), and dead spermatozoa with disaggregated membrane (SYBR-14 negative/PI positive).

Mitochondrial Membrane Potential Analysis. The lipophilic cation JC-1 (Molecular Probes, Eugene, OR) was used to assess sperm mitochondrial membrane potential. Aliquots of semen were diluted with buffer B to approximately $2.5 \times 10^{6}$ spermatozoa $/ \mathrm{mL}$. An 
aliquot of $300 \mu \mathrm{L}$ of the diluted semen was stained with $1.2 \mu \mathrm{L}$ of JC-1 (final stain concentration $12 \mu \mathrm{M}$ ), gently mixed, and incubated at $37^{\circ} \mathrm{C}$ in a water bath in the dark for $40 \mathrm{~min}$. Green fluorescence was detected using an FL1 filter (530/28 nm), and orange fluorescence was measured using a FL2 band-pass filter $(575 / 26 \mathrm{~nm})$. Compensation values were $96.4 \%$ for FL1-FL2 and $39.6 \%$ FL2-FL1.

After the cytometric analysis, sperm samples were classified as proportions in 2 groups: spermatozoa with high respiratory activity (JC-1 positive/orange fluorescence) and spermatozoa with low respiratory activity (JC-1 negative/green fluorescence).

SCSA. This assay (Evenson et al., 2002) used the metachromatic fluorochrome acridine orange to assess sperm chromatin integrity. The metachromatic shift from green fluorescence (stable, double-stranded DNA) to red fluorescence (denatured, single-stranded DNA) in damaged chromatin was quantified by flow cytometry. The DNA fragmentation index (\%DFI) was calculated as the ratio of sperm cells with denatured single-stranded DNA to total DNA (single-stranded + double-stranded DNA). Briefly, samples of thawed semen $(20 \mu \mathrm{L})$ were mixed with an equal volume of buffer containing Tris, $\mathrm{NaCl}$, and EDTA (TNE buffer; $0.15 M \mathrm{NaCl}, 0.01 M$ Tris-HCl, $1 \mathrm{~m} M$ EDTA; pH 7.4), and were immediately submerged in liquid nitrogen. These samples were stored at $-80^{\circ} \mathrm{C}$ until analysis. They were thawed on crushed ice and further diluted with TNE buffer $(1: 10, \mathrm{vol} / \mathrm{vol})$. The spermatozoa were mixed with $200 \mu \mathrm{L}$ of an acid-detergent solution $(0.17 \%$ Triton X-100, $0.15 \mathrm{~mol} / \mathrm{L} \mathrm{NaCl}$, and $0.08 \mathrm{~mol} / \mathrm{L} \mathrm{HCl}$; $\mathrm{pH}$ 1.2). Thirty seconds later, the spermatozoa were stained with $600 \mu \mathrm{L}$ of acridine orange (final concentration $4 \mu \mathrm{g} / \mathrm{mL}$ ) and analyzed within 3 to $5 \mathrm{~min}$. In samples run on the LSR cytometer, green fluorescence was detected with an FL1 band pass filter $(530 / 28 \mathrm{~nm})$ and red fluorescence with an FL3 long-pass filter $(>670$ $\mathrm{nm})$. For samples run on the FACStarPLUS, green fluorescence was detected with an FL1 band pass filter (530/30 nm) and red fluorescence with an FL3 bandpass filter $(660 / 20 \mathrm{~nm})$. Compensation was not used. Calculation of \%DFI was performed.

Measurement of ROS. Hydroethidine (HE; Molecular Probes Inc.) and 2',7'-dichlorodihydrofluorescein diacetate (DCFDA; Sigma-Aldrich, Stockholm, Sweden) were used to detect superoxide and hydrogen peroxide $\left(\mathrm{H}_{2} \mathrm{O}_{2}\right)$, respectively. Hoechst 33258 (HO; Sigma-Aldrich) was added to permit the simultaneous differentiation of living and dead cells. The sperm concentration was adjusted to $2 \times 10^{6}$ spermatozoa/ $\mathrm{mL}$ with buffer $\mathrm{B}$, and $300 \mu \mathrm{L}$ of diluted sample was stained with $9 \mu \mathrm{L}$ of $\mathrm{HE}$ (final stain concentration 1.2 $\mu M$ ), $9 \mu \mathrm{L}$ of DCFDA (final stain concentration 60 $\mu M$ ), and $9 \mu \mathrm{L}$ of $\mathrm{HO}$ (final stain concentration 1.2 $\mu M)$. Samples were gently mixed and incubated at $37^{\circ} \mathrm{C}$ in a water bath in the dark for 30 min before cytometric analysis. The ROS analysis method was modified from Guthrie and Welch (2006) by using HO instead of YoPro-1 as an independent analysis of living spermatozoa. Excitation was achieved with an argon-ion laser (488 $\mathrm{nm})$ and a helium-cadmium laser $(325 \mathrm{~nm})$. Detection of green fluorescence was measured with an FL1 pass filter $(530 / 28 \mathrm{~nm})$, red fluorescence was measured using an FL3 long-pass filter (>670 nm), and blue fluorescence was detected in FL4 with a band-pass filter $(510 / 20 \mathrm{~nm})$. Compensation was not used. After gating for spermatozoa as described above, the FL1/FL4 and FL2/FL4 dotplots were used to identify and quantify the following categories: live (HO negative), superoxide negative; live (HO negative), superoxide positive; dead (HO positive), superoxide positive; live (HO negative), $\mathrm{H}_{2} \mathrm{O}_{2}$ negative; live (HO negative), $\mathrm{H}_{2} \mathrm{O}_{2}$ positive; dead ( $\mathrm{HO}$ positive), $\mathrm{H}_{2} \mathrm{O}_{2}$ negative; and dead (HO positive), $\mathrm{H}_{2} \mathrm{O}_{2}$ positive.

\section{Statistical Analysis}

Statistical analysis was performed using SAS software (version 9.3; SAS Institute Inc., Cary, NC). In total, 60 records were used. Mean values and standard deviations for the sperm kinematic variables and sperm quality parameters were calculated. Data were statistically analyzed by ANOVA (PROC GLM, SAS Inst. Inc.) according to a statistical model that included fixed effects of country, season, the interaction between country and season, and bull nested within country. Residual correlations were calculated between the variables of interest. $P$-values $<0.01$ were regarded as significant.

\section{RESULTS}

\section{Sperm Quality}

The mean values of various sperm characteristics and climate conditions are shown in Tables 1 and 2. These values were reported separately for the study on Holstein and Swedish Red bulls in Sweden (Valeanu et al., 2015) and for the Holstein bulls in the north of Spain (SabésAlsina et al., 2017). Differences among seasons were found for VAP, STR, BCF, \%DFI, and live superoxide negative $(P<0.05)$. We detected differences between the 2 countries $(P<0.01)$ in all parameters except MT, MP, \%DFI, live superoxide positive, and dead $\mathrm{H}_{2} \mathrm{O}_{2}$ positive. There was an interaction between season and country for VAP, VCL, VSL, and live $\mathrm{H}_{2} \mathrm{O}_{2}$-producing spermatozoa $(P<0.05)$. Significant differences were 
CORRELATIONS BETWEEN CLIMATE AND SPERM QUALITY

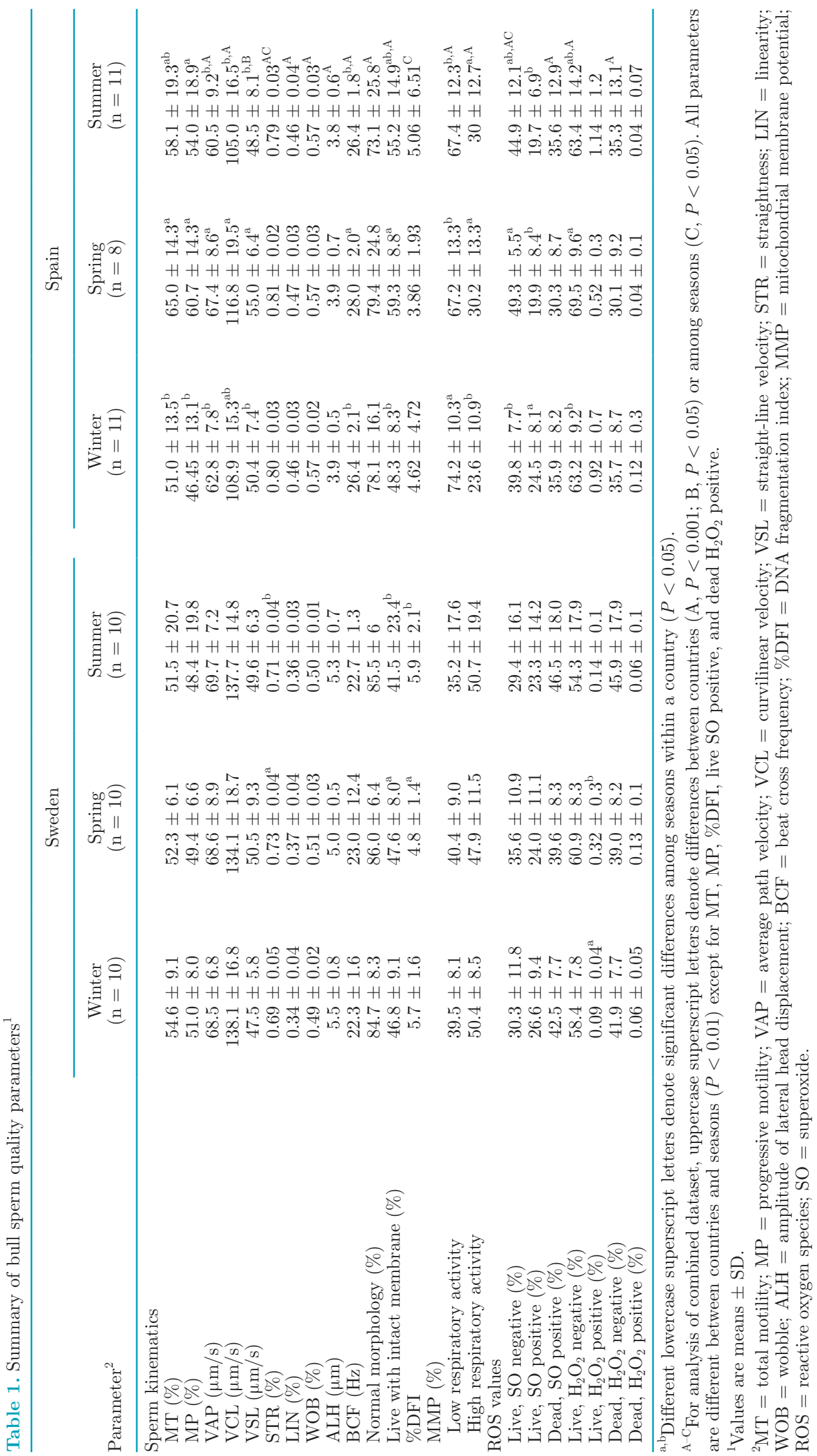


Table 2. Climatic conditions at specific time points relative to month of collection in Sweden and Spain $^{1}$

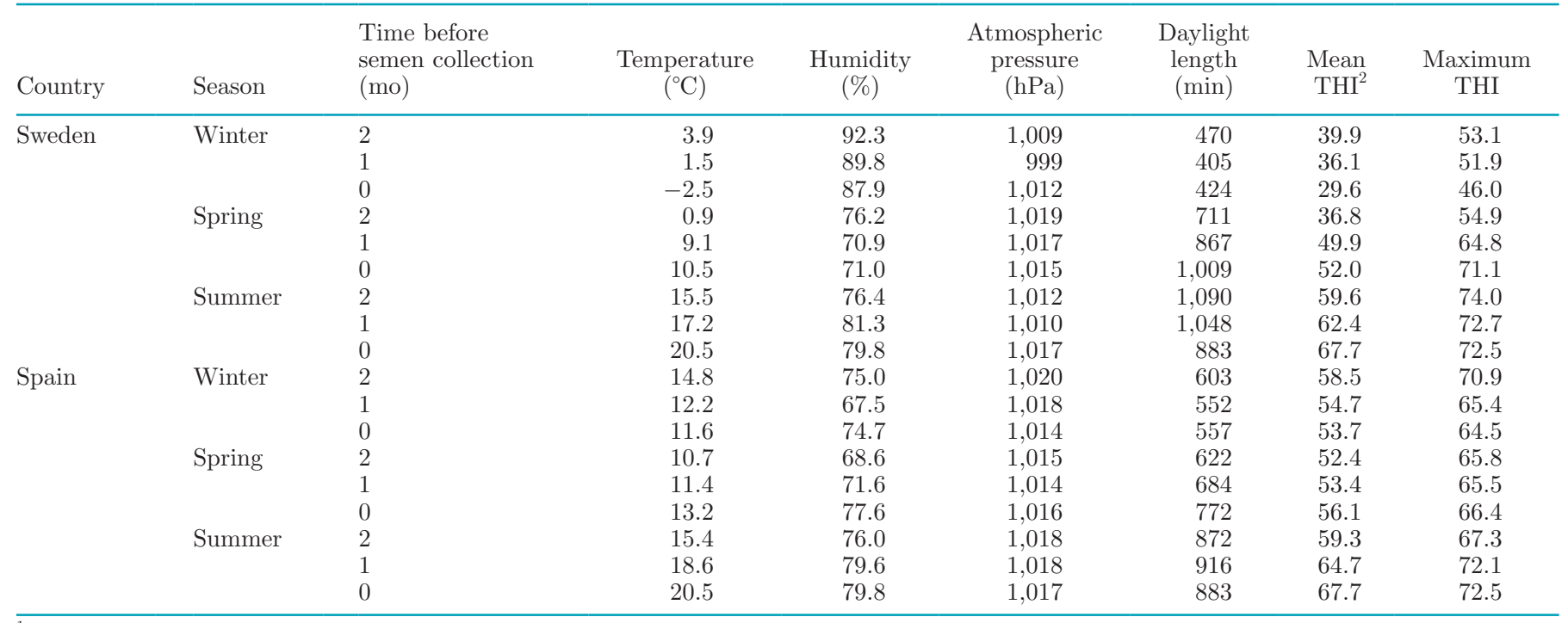

${ }^{1}$ Swedish data were from Swedish Meteorological and Hydrological Institute (Linköping, Sweden); Spanish data were from Agencia Estatal de Meteorología (Gijón, Spain) and the Instituto Geográfico Nacional (Madrid, Spain). Modified from Valeanu et al. (2015) and Sabés-Alsina et al. (2017).

${ }^{2} \mathrm{THI}=$ temperature-humidity index.

observed for all climate factors between countries and among seasons, with a significant interaction for each $(P<0.01)$.

Interrelationships between climate factors are shown in Table 3: climate factors differed according to the time point before semen collection; for example, day length was correlated with most of the other climate factors in the same month as semen collection and 2 mo before semen collection, but not 1 mo before semen collection. Maximum THI was related to all factors except humidity in the same month as semen collection and 2 mo before semen collection, but was only related to temperature and atmospheric pressure 1 mo before semen collection. We detected an interaction between

Table 3. Interrelationships between climate factors (practical correlation coefficients)

\begin{tabular}{|c|c|c|c|c|c|c|}
\hline Item & Temperature & Humidity & $\begin{array}{c}\text { Atmospheric } \\
\text { pressure }\end{array}$ & $\begin{array}{l}\text { Day } \\
\text { length }\end{array}$ & $\begin{array}{l}\text { Mean } \\
\mathrm{THI}^{1}\end{array}$ & $\begin{array}{c}\text { Maximum } \\
\text { THI }\end{array}$ \\
\hline \multicolumn{7}{|c|}{ Same month as semen collection } \\
\hline Temperature & & & & $0.55 * * *$ & $0.999^{* * *}$ & $0.69^{* * *}$ \\
\hline Humidity & $-0.63^{* * *}$ & & $-0.35^{*}$ & $0.47^{* *}$ & & \\
\hline Atmospheric pressure & & $-0.35^{*}$ & & $-0.49^{* *}$ & & $-0.48 * *$ \\
\hline Day length & $0.55^{* * *}$ & $0.47^{* *}$ & $-0.49^{* *}$ & & $0.56^{* * *}$ & $0.52^{* * *}$ \\
\hline Mean THI & $0.999^{* * *}$ & & & $0.56^{* * *}$ & & $0.69 * * *$ \\
\hline Maximum THI & $0.69^{* * *}$ & & $-0.48^{* *}$ & $0.52^{* * *}$ & $0.69^{* * *}$ & \\
\hline \multicolumn{7}{|c|}{1 mo before semen collection } \\
\hline Temperature & & $0.63^{* *}$ & $-0.69 * * *$ & & & $0.57^{* * *}$ \\
\hline Humidity & $0.63^{* * *}$ & & & $0.47^{* *}$ & & \\
\hline Atmospheric pressure & $-0.69 * * *$ & & & & & $-0.41 * *$ \\
\hline Day length & & $0.47^{* *}$ & & & & \\
\hline Mean THI & $-0.32^{*}$ & & & & & \\
\hline Maximum THI & $0.57 * * *$ & & $-0.41^{* *}$ & & & \\
\hline \multicolumn{7}{|c|}{2 mo before semen collection } \\
\hline Temperature & & & $-0.44^{* *}$ & $0.58^{* * *}$ & $0.997^{* * *}$ & $0.78^{* * *}$ \\
\hline Humidity & & & $0.72^{* * *}$ & & & \\
\hline Atmospheric pressure & $-0.44^{* *}$ & $0.72^{* * *}$ & & $-0.57^{* * *}$ & $-0.46^{* *}$ & $-0.71^{* * *}$ \\
\hline Day length & $0.58^{* * *}$ & & $-0.57 * * *$ & & $0.62^{* * *}$ & $0.77^{* * *}$ \\
\hline Mean THI & $0.997 * * *$ & & $-0.46^{* *}$ & $0.62^{* * *}$ & & $0.79^{* * *}$ \\
\hline Maximum THI & $0.78^{* * *}$ & & $-0.71^{* * *}$ & $0.77^{* * *}$ & $0.79^{* * *}$ & \\
\hline
\end{tabular}

${ }^{1} \mathrm{THI}=$ temperature-humidity index.

${ }^{*} P<0.05 ;{ }^{* *} P<0.01 ;{ }^{* * *} P<0.001$. 
country and season, indicating that the climate effects were not the same in the 2 countries.

\section{Correlations of Sperm Quality with Climate in the Same Month as Sperm Collection}

There were few significant correlations of kinematics and climate in the same month as semen collection. Day length was negatively correlated with STR $(\mathrm{r}=$ $-0.33 ; P<0.05)$ and LIN $(\mathrm{r}=0.37 ; P<0.05)$; mean THI was negatively correlated with STR $(\mathrm{r}=-0.37 ; P$ $<0.05)$. No other significant relationships were found between the remaining kinematics and climate factors at this time point.

Similarly, few significant correlations were found between sperm quality parameters and climate factors in the same month as semen collection, with \%DFI and proportion of live spermatozoa producing $\mathrm{H}_{2} \mathrm{O}_{2}$ being the factors affected. The \%DFI was correlated with temperature $(\mathrm{r}=0.46 ; P<0.05)$ and maximum THI $(\mathrm{r}=0.71 ; P<0.001)$. The proportion of live, $\mathrm{H}_{2} \mathrm{O}_{2^{-}}$ producing spermatozoa was negatively correlated with day length $(\mathrm{r}=-0.49 ; P<0.01)$ and positively correlated with atmospheric pressure $(\mathrm{r}=0.48 ; P<0.01)$ and maximum THI $(\mathrm{r}=0.46 ; P<0.01)$.

\section{Correlations of Sperm Quality with Climate One Month Before Sperm Collection}

Significant correlations were seen between humidity and the following kinematics: VCL $(\mathrm{r}=0.46 ; P<$ $0.01)$, LIN ( $\mathrm{r}=-0.37 ; P<0.05)$, WOB $(\mathrm{r}=-0.41$; $P<0.05)$, and ALH $(\mathrm{r}=0.5 ; P<0.01)$. In addition, day length was significantly correlated with LIN ( $\mathrm{r}=$ $-0.36 ; P<0.05)$ and WOB $(\mathrm{r}=-0.38 ; P<0.05)$. No other significant relationships were observed between the remaining kinematic features and climate factors at this time point. Regarding other sperm quality parameters, \%DFI was correlated with temperature $(\mathrm{r}=0.33$; $P<0.05)$ and maximum THI $(\mathrm{r}=0.46 ; P<0.051)$ and negatively correlated with atmospheric pressure $(\mathrm{r}=$ $-0.48 ; P<0.01)$. Normal morphology was negatively correlated with maximum THI $(\mathrm{r}=-0.42 ; P<0.01)$. The proportion of live spermatozoa producing $\mathrm{H}_{2} \mathrm{O}_{2}$ was correlated with maximum THI $(\mathrm{r}=0.34 ; P<0.05)$ and negatively correlated with humidity $(\mathrm{r}=-0.47 ; P$ $<0.01)$. None of the other parameters of sperm quality were correlated with climate factors.

\section{Correlations of Sperm Quality with Climate Two Months Before Sperm Collection}

Regarding kinematics, atmospheric pressure was correlated with LIN $(\mathrm{r}=0.33 ; P<0.05)$ and WOB $(\mathrm{r}=$
$0.36 ; P<0.05)$ and negatively correlated with ALH ( $\mathrm{r}=-0.39 ; P<0.05)$. In addition, VCL was related to temperature $(\mathrm{r}=0.34 ; P<0.05)$ and mean THI ( $\mathrm{r}=0.34 ; P<0.05)$ and negatively correlated with atmospheric pressure $(\mathrm{r}=-0.42 ; P<0.05)$. Mean THI was correlated with VAP $(\mathrm{r}=0.32 ; P<0.05)$.

Significant correlations were seen between climate factors and \%DFI, morphology, and live, $\mathrm{H}_{2} \mathrm{O}_{2}$-producing spermatozoa. The \%DFI was correlated with temperature $(\mathrm{r}=0.44 ; P<0.01)$, day length $(\mathrm{r}=$ $0.41 ; P<0.01)$, mean THI $(\mathrm{r}=0.44 ; P<0.01)$, and maximum THI $(\mathrm{r}=0.71 ; P<0.001)$ and negatively correlated with atmospheric pressure $(\mathrm{r}=-0.45 ; P<$ $0.01)$. Normal morphology was negatively correlated with maximum THI $(\mathrm{r}=-0.48 ; P<0.01)$. The proportion of live spermatozoa producing $\mathrm{H}_{2} \mathrm{O}_{2}$ was correlated with maximum THI $(\mathrm{r}=0.34 ; P<0.05)$ and negatively correlated with temperature $(\mathrm{r}=-0.49 ; P$ $<0.01)$ and humidity $(\mathrm{r}=-0.47 ; P<0.01)$. None of the other parameters of sperm quality were correlated with climate factors.

\section{Correlations Within Sperm Quality Parameters}

Significant correlations among sperm quality parameters are shown in Table 4. The pattern of correlations was similar at all time points, differing only in the value of the correlation coefficient and not in the level of significance; therefore only one figure is shown for all time points. Normal morphology and \%DFI were negatively correlated, as were mitochondrial activity and ROS production. Mitochondrial activity was correlated with motility and membrane integrity.

\section{DISCUSSION}

This retrospective study investigated possible correlations between mean values of climate factors recorded at several points (during the month of sperm collection and up to 2 mo previously) and sperm quality parameters of frozen-thawed bovine semen collected in Sweden and northern Spain. There were methodological differences between the 2 sets of samples, so differences between countries in this case are not only an effect of geographical location. The results showed that sperm quality parameters (in terms of morphology, \%DFI, metabolic activity, and ROS) were correlated with exposure of animals to specific climate factors before or during the month of semen collection. Some kinematics were also apparently correlated with climate factors but it should be noted that there was a significant interaction between country and season for VAP, VCL, and VSL; thus, interpretation of a possible "climate" effect should be treated with caution. The correlations 


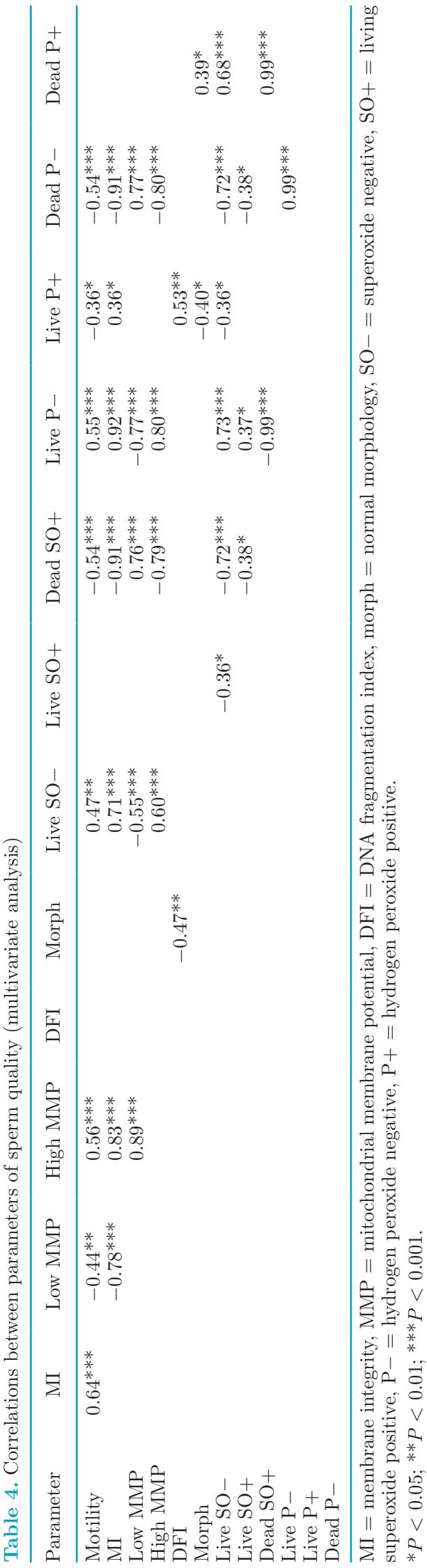

between some of the climate factors should be taken into account when interpreting the possible effects of climate on sperm quality; for example, daylight length and temperature were correlated.

This study demonstrated that the effect of specific climate factors on sperm quality at a specified time differed, presumably according to the stage of spermatogenesis at the time. Sperm kinematics were affected by daylight length and mean THI during the same month of semen collection, by humidity and daylight length 1 mo before semen collection, and by atmospheric pressure 2 mo before semen collection. Furthermore, \%DFI was affected by temperature and maximum THI in the same month as semen collection, by humidity and maximum THI 1 mo before semen collection, and by all climate factors except humidity 2 mo before semen collection. Normal morphology was affected only by THI 1 and 2 mo before semen collection. Production of $\mathrm{H}_{2} \mathrm{O}_{2}$ was related to maximum THI at all time points; to temperature and atmospheric pressure in the same month as semen collection and 2 mo before semen collection; and to humidity 1 mo before semen collection.

During the early stages of spermatogenesis, new germ cells (such as pachytene and diplotene spermatocytes) are vulnerable to high testicular temperatures because of the high mitotic activity within this organ (Parkinson, 1987; Januskauskas et al., 1995). The expected affected mechanisms are cell apoptosis and autophagy, DNA damage (altered synapsis and strand breaks), generation of ROS, and reduction of sperm motility (Januskauskas et al., 1995) that can be seen in the ejaculates around 2 mo later. Our results for \%DFI and $\mathrm{H}_{2} \mathrm{O}_{2}$ production are consistent with these previous results.

Regarding our methodology for ROS evaluation, Huo et al. (2002) found a correlation of 0.996 between viability measurements using SYBR-14/PI and HO in boar spermatozoa, whereas Johannisson et al. (2018) found a lack of correlation between these measurements in stallion spermatozoa. In the present study, there was generally good agreement between the results of these 2 assays, pointing to a difference among species in the applicability of $\mathrm{HO}$ as an indicator of membrane integrity.

During epididymal maturation (late stage of spermatogenesis), the effects of high temperatures on sperm cells are different from those seen in the testis. Sperm quality parameters that could be expected to be affected during this phase are sperm count, ATP concentration, fertilizing capacity, and sperm membrane integrity, seen in the ejaculate around 16 and $23 \mathrm{~d}$ after the heat stress (Januskauskas et al., 1995; Malama et al., 2012). Finally, the possible negative effects of high temperatures on the sperm cells present in the epididymis or the rete testis can be seen in the ejaculate 
around 2 and $9 \mathrm{~d}$ after the heat stress (Januskauskas et al., 1995). We did not see an effect on membrane integrity, but this may have been due to the wider time intervals (same month, $1 \mathrm{mo}$, and $2 \mathrm{mo}$ ) in our study compared with other studies.

Another important finding in the present study was that sperm quality parameters were more likely to be correlated with climate factors measured 2 mo before semen collection than in the same month of semen collection.

The results from this study corroborate the idea that exposure of animals to potentially detrimental conditions, such as heat stress, can compromise spermatogenesis and produce changes in ejaculate characteristics that would be observed between 2 and $4 \mathrm{wk}$ after the negative event (Kastelic et al., 1996; Senger, 1997; Malama et al., 2012). These alterations in sperm quality vary depending on the point during spermatogenesis that the detrimental condition is experienced. Januskauskas et al. (1995) found that scrotal insulation applied to sperm cells present in the epididymis and rete testis (sperm cells obtained between 2 and $9 \mathrm{~d}$ after insulation) did not produce significant differences on the parameters analyzed (motility, morphology, ATP content, viability, and sperm membrane integrity). However, the same scrotal insulation applied during an early stage of spermatogenesis (sperm cells obtained between 30 and $51 \mathrm{~d}$ after insulation) or a late stage of spermatogenesis (sperm cells obtained between 16 and $23 \mathrm{~d}$ after insulation) produced a significant increase of sperm morphology abnormalities and a decrease in motility, sperm membrane integrity, and sperm ATP concentration. In contrast, Kastelic et al. (1997b) argued that the temperature gradient within the scrotum suggests that spermatozoa in the cauda epididymis may be more susceptible to elevated temperature than testicular spermatozoa. Parkinson (1987) observed a quadratic relationship between temperature and abnormal spermatozoa in the ejaculate 1 or 2 mo later. We observed a negative effect of maximum THI on normal morphology 2 and 1 mo before semen collection but no effect on motility or membrane integrity. These differences may be due to the different methodologies; that is, scrotal insulation in the study by Januskauskas et al. (1995) versus no insulation in our study or that of Parkinson (1987).

Some kinematics were correlated with day length and mean THI during the same month of semen collection. However, these kinematics were not correlated with either day length or mean THI 2 mo before sperm collection. These findings are in agreement with those of Malama et al. (2017), who found that sperm samples collected under conditions of high temperature (31.8 \pm $\left.1.4^{\circ} \mathrm{C}\right)$ and humidity $(84.3 \pm 3.8 \%)$ in summer showed altered motility, although those authors did not observe impaired sperm chromatin integrity of ejaculates collected under elevated summer temperatures. They did report an effect of microclimate in the $11 \mathrm{~d}$ before ejaculation on sperm DNA integrity of bulls in Greece (Malama et al., 2012). Bhakat et al. (2014) found that sperm samples collected under conditions of high temperature $\left(31.82^{\circ} \mathrm{C}\right)$ but low humidity $(43.59 \%)$ in summer had a negative effect on motility, sperm concentration, hypo-osmotic swelling test, acrosome integrity, morphology abnormalities, and osmolality.

A possible explanation for the variations in some sperm quality parameters between studies may be due to differences in humidity and therefore differences in the mean THI. Alternatively, husbandry conditions such as stocking density or availability of water may affect the animals' ability to regulate scrotal temperature. Methodological differences in the sperm quality assays performed in the various studies may also contribute to differences in the results obtained. It is possible that mature sperm cells in the ampulla may be less susceptible to the effects of temperature than immature sperm cells in the testes in animals in which the testes are located outside the abdomen. This could explain why mature sperm cells can be stored in the abdomen in animals possessing an ampulla; that is, at body temperature, whereas immature sperm cells in the testes and maturing sperm in the epididymides are stored at a lower temperature in the scrotum.

Overall, values of sperm morphology correlated significantly only with maximum THI. These morphology results are in agreement with those of Parkinson (1987) and Januskauskas et al. (1995). The latter observed that $30 \mathrm{~d}$ after a brief elevation of scrotal temperature, only abnormal head shape was significantly increased among all sperm abnormalities analyzed (acrosome, midpiece, and sperm tail). However, our findings are in contrast to those of another study (Bhakat et al., 2014), which reported that total sperm morphology abnormalities differed according to season, being highest in summer followed by the rainy season, and winter having the fewest abnormalities. Several factors such as age and breed of the animal, level of nutrition, management practice, and so on, as well as severity of the climatic factors, may be responsible for the differences between studies.

Our findings differ from the scrotal insulation studies already cited. These differences could be due to the temperate climatic conditions, which may not have been extreme enough to exert an effect on sperm morphology, or that the physiological mechanisms controlling testicular temperature (e.g., muscle relaxation to allow the testes to hang away from the body or increased vascularization to remove heat) were sufficient to maintain 
normal temperatures within the scrotum despite the increased temperature and humidity (Kastelic et al., 1997a; Durairajanayagam et al., 2015). Similar findings have been observed in stallions exercised in hot climates: testicular temperature was maintained and no effect on sperm quality was observed, despite an increase in body temperature (Mawyer et al., 2012). Alternatively, scrotal insulation may not be an appropriate model to study climatic factors because normal physiological mechanisms to control testicular temperature are completely overcome.

\section{CONCLUSIONS}

The present study confirmed that dairy bull sperm quality is affected by climatic conditions, even in socalled temperate zones. The timing of heat stress during spermatogenesis determines which aspects of sperm quality are likely to be affected. Husbandry conditions for bulls used for semen collection should be adapted to allow the animals' physiological responses for temperature regulation to operate fully so that the effects of increased temperature and humidity can be mitigated. Extremes of temperature and humidity should be avoided.

\section{ACKNOWLEDGMENTS}

The authors thank Viking Genetics (Skara, Sweden) and ASCOL (Gijón, Spain) for providing the straws of frozen semen and Karin Selin-Wretling of the Sperm Laboratory at SLU (Uppsala, Sweden) for the morphology analysis. We thank Sabina Valeanu (Swedish Univeristy of Agricultural Sciences, Uppsala, Sweden) for technical assistance with analyzing some of the samples. The authors gratefully acknowledge the financial support of the Ministerio de Economía y Competitividad and FEDER (AGL2016-79802-P). Maria Sabés-Alsina was supported by a Predoctoral Research Fellowship (PIF) from the Universitat Autònoma de Barcelona and by the Short Term Scientific Mission (STSM) of the Epiconcept COST Action; Jane M. Morrell and Anders Johannisson were funded by the Swedish Research Council for the environment, agricultural sciences and spatial planning (FORMAS, Stockholm, Sweden), project number 221-2010-1241, and the Swedish Farmer's Association (SLF, Stockholm, Sweden), project number 1330039 .

\section{REFERENCES}

Amann, R. P., and J. O. Almquist. 1962. Reproductive capacity of dairy bulls. VIII. Direct and indirect measurement of testicular sperm production. J. Dairy Sci. 45:774-781. https://doi.org/10 .3168/jds.S0022-0302(62)89487-9.

Belda, M., E. Holtanova, T. Halenka, and J. Kalvova. 2014. Climate classification revisited: From Köppen to Trewartha. Clim. Res. 59:1-13.

Bhakat, M., T. K. Mohanty, A. K. Gupta, and M. Abdullah. 2014. Effect of season on semen quality of crossbred (Karan Fries) bulls. Adv. Anim. Vet. Sci. 2:632-637.

Blackshaw, J. K., and A. W. Blackshaw. 1994. Heat stress in cattle and the effect of shade on production and behaviour: A review. Aust. J. Exp. Agric. 34:285-295.

Bohmanova, J., I. Misztal, and J. B. Cole. 2007. Temperature-humidity indices as indicators of milk production losses due to heat stress. J. Dairy Sci. 90:1947-1956. https://doi.org/10.3168/jds.2006-513.

Brown, B. W. 1994. A review of nutritional influences on reproduction in boars, bulls and rams. Reprod. Nutr. Dev. 34:89-114.

Durairajanayagam, D., A. Agarwal, and C. Ong. 2015. Causes, effects and molecular mechanisms of testicular heat stress. Reprod. Biomed. Online 30:14-27. https://doi.org/10.1016/j.rbmo.2014.09 .018 .

Evenson, D.P., K. L. Larson, and L. K. Jost. 2002. Sperm chromatin structure assay: Its clinical use for detecting sperm DNA fragmentation in male infertility and comparisons with other techniques. J. Androl. 23:25-43.

Gantner, V., P. Mijić, K. Kuterovac, D. Solić, and R. Gantner. 2011. Temperature-humidity index values and their significance on the daily production of dairy cattle. Mljekarstvo 61:56-63.

García-Ispierto, I., F. López-Gatius, P. Santolaria, J. L. Yániz, C. Nogareda, M. López-Béjar, and F. De Rensis. 2006. Relationship between heat stress during the peri-implantation period and early fetal loss in dairy cattle. Theriogenology 65:799-807. https://doi .org/10.1016/j.theriogenology.2005.06.011.

Guthrie, H. D., and G. R. Welch. 2006. Determination of intracellular reactive oxygen species and high mitochondrial membrane potential in Percoll-treated viable boar sperm using fluorescenceactivated flow cytometry. J. Anim. Sci. 84:2089-2100. https://doi . org $/ 10.2527 /$ jas. $2005-766$.

Hansen, P. J. 2009. Effects of heat stress on mammalian reproduction. Philos. Trans. R. Soc. B Biol. Sci. 364:3341-3350. https://doi.org/ 10.1098/rstb.2009.0131.

Huo, L. J., X. H. Ma, and Z. M. Yang. 2002. Assessment of sperm viability, mitochondrial activity, capacitation and acrosome intactness in extended boar semen during long-term storage. Theriogenology 58:1349-1360. https://doi.org/10.1016/S0093-691X(02)00953-6.

Januskauskas, A., J. Gil, H. Rodríguez-Martínez, L. Söderquist, and N. Lundeheim. 1995. Effects of a brief elevation of scrotal temperature on the post-thaw viability of bull semen. Reprod. Domest. Anim. 30:271-277. https://doi.org/10.1111/j.1439-0531.1995 .tb00624.x.

Johannisson, A., M. I. Figueiredo, Z. Al-Kass, and J. M. Morrell. 2018. Simultaneous evaluation of superoxide content and mitochondrial membrane potential in stallion semen samples provides additional information about sperm quality. Anim. Reprod. Sci. 192:290-297. https://doi.org/10.1016/j.anireprosci.2018.03.030.

Kastelic, J. P., R. B. Cook, and G. H. Coulter. 1997a. Scrotal/testicular thermoregulation and the effects of increased testicular temperature in the bull. Vet. Clin. North Am. Food Anim. Pract. 13:271-282.

Kastelic, J. P., R. B. Cook, and G. H. Coulter. 1997b. Contribution of the scrotum, testes, and testicular artery to scrotal/testicular thermoregulation in bulls at two ambient temperatures. Anim. Reprod. Sci. 45:255-261.

Kastelic, J. P., R. B. Cook, G. H. Coulter, and R. G. Saacke. 1996 Insulating the scrotal neck affects semen quality and scrotal/testicular temperatures in the bull. Theriogenology 45:935-942.

Malama, E., W. E. Kiossis, T. Theodosiou, C. Boscos, and H. Bollwein. 2012. Lag effect of microclimatic conditions on DNA integrity of frozen-thawed bovine sperm. Anim. Reprod. Sci. 136:33-41.

Malama, E., Y. Zeron, F. Janett, M. Siuda, Z. Roth, and H. Bollwein. 2017. Use of computer-assisted sperm analysis and flow cytometry 
to detect seasonal variations of bovine semen quality. Theriogenology 87:79-90. https://doi.org/10.1016/j.theriogenology.2016.08 .002 .

Mawyer, J. D., C. A. Cavinder, M. M. Vogelsang, D. H. Sigler, C. C. Love, S. P. Brinsko, T. L. Blanchard, D. D. Varner, C. E. Arnold, S. Teague, and R. K. Gordon. 2012. Thermoregulation of the testicle in response to exercise and subsequent effects on semen characteristics of stallions. J. Anim. Sci. 90:2532-2539. https://doi .org/10.2527/jas.2011-4543.

Mocé, E., and J. K. Graham. 2008. In vitro evaluation of sperm quality. Anim. Reprod. Sci. 105:104-118. https://doi.org/10.1016/j .anireprosci.2007.11.016.

Morrell, J. M., A.-M. Dalin, and H. Rodríguez-Martínez. 2008. Prolongation of stallion sperm survival by centrifugation through coated silica colloids: A preliminary study. Anim. Reprod. 5:121-126.

Morrell, J. M., and H. Rodríguez-Martínez. 2009. Biomimetic techniques for improving sperm quality in animal breeding: A review. Open Androl. J. 1:1-9.

Parkinson, T. J. 1987. Seasonal variations in semen quality of bulls: Correlations with environmental temperatures. Vet. Rec. 120:479 482 .
Sabés-Alsina, M., A. Johannisson, N. Lundeheim, M. Lopez-Bejar, and J. M. Morrell. 2017. Effects of season on bull sperm quality in thawed samples in northern Spain. Vet. Rec. 180:251-257.

Senger, P. L. 1997. Pathways to Pregnancy and Parturition. Current Conceptions Inc., Redmond, OR.

Snoj, T., S. Kobal, and G. Majdic. 2013. Effects of season, age, and breed on semen characteristics in different Bos taurus breeds in a 31-year retrospective study. Theriogenology 79:847-852. https:// doi.org/10.1016/j.theriogenology.2012.12.014.

Valeanu, S., A. Johannisson, N. Lundeheim, and J. M. Morrell. 2015. Seasonal variation in sperm quality parameters in Swedish red dairy bulls used for artificial insemination. Livest. Sci. 173:111118. https://doi.org/10.1016/j.livsci.2014.12.005.

Verrati, V., C. Di Giulio, A. D'Angeli, A. Tafuri, S. Francavilla, and F. Pelliccione. 2016. Sperm forward motility is negatively affected by short-term exposure to altitude hypoxia. Andrologia 48:800-806.

Vogler, C. J., J. H. Bame, M. L. DeJamette, M. L. McGilliard, and R. G. Saacke. 1993. Effects of elevated testicular temperature on morphology characteristics of ejaculated spermatozoa in the bovine. Theriogenology 40:1207-1219. 